\title{
Mathematical Modeling \\ of Aluminum Reduction Cell MHD Stability
}

\author{
Nadejda P. Savenkova ${ }^{a}$, Andrei Yu. Mokina, \\ Nellya S. Udovichenko a and Artem A. Pianykh*b \\ ${ }^{a}$ Lomonosov Moscow State University \\ Moscow, Russian Federation \\ ${ }^{b}$ Siberian Federal University \\ Krasnoyarsk, Russian Federation
}

Received 24.01.2020, received in revised form 02.02.2020, accepted 18.03.2020

Abstract. The paper describes a mathematical model of magnetic hydrodynamics and heat transfer in an aluminum electrolyzer. The model takes into account three phases: gas, electrolyte and metal, and investigates their interaction. Mathematical modeling of the dynamics of the aluminumelectrolyte interface is carried out depending on the potential distribution over the anode for the Soderberg electrolyzer and the multi-anode electrolyzer. A numerical study made it possible to conclude that the Soderberg electrolyzer is less MHD-stable than a multi-anode electrolyzer with burnt anodes.

Calculations of MHD stability are carried out when changing the shape of the working space of the bath for various forms of accretion and skull. The interface between the electrolyte-metal media and the boundary of the reverse oxidation zone, which is determined by the spatial distribution of the gas phase, were calculated. The calculations make it possible to accurately predict the development of MHD instability in the bath under various conditions of the process, which minimizes the loss of metal current efficiency.

Keywords: MHD stability, Soderberg electrolyzer, control volume method.

Citation: Savenkova N.P., Mokin A.Yu., Udovichenko N.S., Pianykh A.A. Mathematical modeling of aluminum reduction cell MHD stability, J. Sib. Fed. Univ. Eng. \& Technol., 2020, 13(2), 243-253. DOI: 10.17516/1999-494X-0211

(C) Siberian Federal University. All rights reserved

This work is licensed under a Creative Commons Attribution-NonCommercial 4.0 International License (CC BY-NC 4.0).

* Corresponding author E-mail address: piartex@gmail.com 


\title{
Математическое моделирование
}

\section{МГД-стабильности алюминиевого электролизера}

\author{
Н.П. Савенкова ${ }^{\mathrm{a}}$, А.Ю. Мокин ${ }^{\mathrm{a}}$, \\ Н.С. Удовиченко ${ }^{a}$, А.А. Пьяных ${ }^{\tilde{0}}$ \\ ${ }^{a}$ Московский государственный университет \\ им. М.В. Ломоносова \\ Российская Федерачия, Москва \\ ${ }^{6}$ Сибирский федеральный университет \\ Российская Федераиия, Красноярск
}

Аннотация. В работе описана математическая модель магнитной гидродинамики и теплообмена в алюминиевом электролизере. В модели учитывают три фазы: газ, электролит и металл, исследуют их взаимодействие. Проведено математическое моделирование динамики границы раздела сред алюминий-электролит в зависимости от распределения потенциала по аноду для электролизера Содерберга и многоанодного электролизера. Проведенное численное исследование позволило сделать вывод о том, что электролизер Содерберга менее МГДстабилен, чем многоанодный электролизер с обожженными анодами.

Выполнены расчеты МГД-стабильности при изменении формы рабочего пространства ванны для различных форм настыли и гарнисажа. Была рассчитана граница раздела сред электролитметалл и граница зоны обратного окисления, которая определяется пространственным распределением газовой фазы. Расчеты позволяют достаточно точно прогнозировать развитие МГД-нестабильности в ванне при различных условиях проведения технологического процесса, что минимизирует потери выхода металла по току.

Ключевые слова: МГД-стабильность, электролизер Содерберга, метод контрольного объема.

Цитирование: Савенкова, Н.П. Математическое моделирование МГД-стабильности алюминиевого электролизера Н.П. Савенкова, А.Ю. Мокин, Н.С. Удовиченко, А.А. Пьяных // Журн. Сиб. федер. ун-та. Техника и технологии, 2020. 13(2). C. 243-253. DOI: 10.17516/1999-494X-0211

\section{Введение}

Представленная модель может быть использована для анализа МГД-стабильности электролизера при его режимных и конструктивных изменениях. Основное преимущество предложенной модели заключается в комплексном подходе при моделировании химических и физических процессов, протекающих в электролизере, а также в их взаимосвязи. В разработанной новой трехмерной модели динамики МГД-стабильности исследуется взаимодействие трех фаз: газовой, которая возникает в процессе электролиза алюминия, собственно электролита и жидкого металла. Модель позволяет определить границы раздела этих сред и достаточно точно прогнозировать развитие МГД-нестабильности в ванне при различных условиях проведения технологического процесса. Проведенные численные эксперименты позволили подтвердить предположение о том, что электролизер Содерберга является менее МГД-стабильным по сравнению с многоанодным электролизером. 


\section{1. Математическая модель динамики изменения границ сред}

Предлагается трехмерная трехфазная (алюминий, электролит и газ) математическая модель, в основе которой находится многофазный подход к описанию среды. В представленной математической модели во взаимосвязи учтены гидродинамические, электромагнитные, тепловые и электрохимические процессы, протекающие в алюминиевом электролизере. В соответствии с общей теорией многофазных сред предполагается, что в гетерогенной смеси каждая фаза занимает лишь часть элементарного объема [1], в связи с этим вводятся объемные доли $\alpha_{m}=\alpha_{m}(x, y, z, t)(m=1,2,3)$ каждой фазы в общем объеме смеси. При этом выполняется балансное соотношение:

$$
\alpha_{1}+\alpha_{2}+\alpha_{3}=1 \text {. }
$$

В каждой точке объема, занятого смесью, рассматриваются следующие параметры смеси: скорости компонент $\boldsymbol{v}_{m}=\left(u_{m}, v_{m}, w_{m}\right)$, давление $p$, напряженность магнитного поля $\boldsymbol{H}=\left(H_{x}, H_{y}, H_{z}\right)$, плотность электрического тока $\boldsymbol{j}=\left(j_{x}, j_{y}, j_{z}\right)$ и температура $T$. Все вышеперечисленные величины являются основными неизвестными математической модели.

Закон сохранения массы каждой фазы описывается уравнением:

$$
\frac{\partial \alpha_{\mathrm{m}} \rho_{\mathrm{m}}}{\partial \mathrm{t}}+\nabla \cdot\left(\alpha_{\mathrm{m}} \rho_{\mathrm{m}} v_{m}\right)=J_{\mathrm{m}}
$$

где $\rho_{m}$ - плотность $m$-й фазы, которая постоянна; $J_{m}$ - источник (сток) вещества $m$-й фазы за счет химических реакций. Учитывая неоднородность химических реакций в пространстве ванны справедливо следующее:

- для фазы алюминия $J_{1}$ - функция, зависящая от его концентрации, равная нулю везде, кроме границы раздела фаз алюминий - электролит;

- для фазы газа $J_{3}$ - функция, зависящая от его концентрации, равная нулю везде, кроме области под анодами;

- для фазы электролита $J_{2}=-J_{1}$ на границе раздела фаз алюминий - электролит, $J_{2}=-J_{3}$ в области под анодами [1-2].

Уравнение движения для $m$-й компоненты смеси имеет вид:

$$
\begin{gathered}
\frac{\partial \alpha_{m} \boldsymbol{v}_{m}}{\partial t}+\nabla \cdot \boldsymbol{v}_{m}\left(\alpha_{m} \rho_{m} \overrightarrow{\boldsymbol{v}_{m}}\right)=-\frac{\alpha_{m}}{\rho_{m}} \nabla p+\alpha_{m} \boldsymbol{g}+\alpha_{m} \frac{\mu_{m}}{\rho_{m}} \Delta \boldsymbol{v}_{m}+ \\
+\sum_{m \neq n, n=1,2,3} \alpha_{n} \frac{\alpha_{m}}{\rho_{m}} K_{n m}\left(\boldsymbol{v}_{m}-\boldsymbol{v}_{n}\right)+\frac{\alpha_{m}}{\rho_{m}}\left(\mu_{0}[\boldsymbol{j} \times \boldsymbol{H}]\right), m=1,2,3,
\end{gathered}
$$

где $K_{n m}-$ средневзвешенный коэффициент вязкости между n-й и т-й фазами, т.е. считаем, что $K_{m n}=\alpha_{m} \mu_{m}+\alpha_{n} \mu_{n}, \mu_{m}, \mu_{m}-$ динамическая вязкость (константа), [Па $\cdot$ с] [1].

Первое и третье слагаемые в правой части (3) - дивергенция тензора напряжений фаз, второе слагаемое описывает действие гравитационных сил, четвертое - передачу импульса между компонентами смеси, пятое - влияние электромагнитных сил (силы Лоренца), учитывая, что плотности сред считаются постоянными.

Граничными условиями являются условия прилипания на границе области

$$
-245-
$$




$$
\left.\boldsymbol{v}_{m}\right|_{\Gamma}=0, m=1,2,3
$$

Для определения давления используем следующее уравнение:

$$
\begin{gathered}
\nabla \cdot\left(\sum_{m=1,2,3}\left(\frac{\alpha_{m}}{\rho_{m}} \nabla p\right)\right)=\nabla \cdot\left(\sum _ { m = 1 , 2 , 3 } \left(-\nabla \cdot \boldsymbol{v}_{m}\left(\alpha_{m} \boldsymbol{v}_{m}\right)+\alpha_{m} \frac{\mu_{m}}{\rho_{m}} \Delta \boldsymbol{v}_{m}+\right.\right. \\
\left.\left.+\alpha_{m} \boldsymbol{g}+\frac{\alpha_{m}}{\rho_{m}}\left(\mu_{0}[\boldsymbol{j} \times \mathbf{H}]\right)+\sum_{m \neq n, n=1,2,3} \alpha_{n} \frac{\alpha_{m}}{\rho_{m}} K_{n m}\left(\boldsymbol{v}_{m}-\boldsymbol{v}_{n}\right)\right)\right)
\end{gathered}
$$

полученное путем сложения уравнений закона сохранения импульса (3) для трех фаз с учетом (1), (2).

В основе математического описания электродинамических процессов, протекающих в электролизной ванне, лежит система уравнений Максвелла. Параметры электромагнитного поля определяются следующими соотношениями:

$$
\boldsymbol{E}=-\nabla \phi, \nabla \cdot\left(\sum_{m=1,2,3}\left(\alpha_{m} \sigma_{m}\right) \nabla \phi\right)=\nabla \cdot\left(\mu_{0}\left[\boldsymbol{v}_{m}, \mathbf{H}\right] \sum_{m=1,2,3}\left(\alpha_{m} \sigma_{m}\right)\right),
$$

здесь $\sigma_{m}$ - электрическая проводимость; $\phi$ - потенциал электрического поля.

Основные молекулы и ионы, участвующие в химических реакциях, представлены в табл. 1.

Движение ионов описывает уравнение Нернста-Планка:

$$
\frac{\partial C_{k}}{\partial t}=D_{k} \nabla \cdot\left(\nabla C_{k}+\frac{z_{k} F}{R T} C_{k} \nabla \phi\right),
$$

где $k$ - порядковый номер иона [3, 4].

Для учета тепловых процессов в объеме ванны используют модель, в которой температура считается скалярной характеристикой смеси и меняется за счет конвекции, диффузии и источника Джоулева тепла. В сделанных предположениях уравнение, которое описывает изменение температуры, выглядит следующим образом:

$$
\frac{\partial \rho T}{\partial t}+\nabla \cdot(\rho T \boldsymbol{v})=\frac{1}{\zeta} \nabla \cdot(\lambda \nabla T)+\frac{1}{\zeta}(\boldsymbol{j}, \boldsymbol{E})
$$

Таблица 1. Ионы, участвующие в химических реакциях

Table 1. Ions involved in chemical reactions

\begin{tabular}{|c|c|c|c|}
\hline № & Ион & Заряд & Знак \\
\hline 1 & $\mathrm{Na}$ & 1 & + \\
\hline 2 & $\mathrm{AlF} F_{4}$ & 3 & - \\
\hline 3 & $\mathrm{AlF}_{6}$ & 1 & - \\
\hline 4 & $F$ & 1 & - \\
\hline 5 & $\mathrm{Al}_{2} \mathrm{O}_{2} F_{4}$ & 2 & - \\
\hline 6 & $\mathrm{Al}_{2} \mathrm{OF}_{6}$ & 2 & \\
\hline
\end{tabular}


где значения величин скорости, теплоемкости, теплопроводности и плотности берут средневзвешенными:

$$
\begin{aligned}
& \boldsymbol{v}=\boldsymbol{v}_{1} \alpha_{1}+\boldsymbol{v}_{2} \alpha_{2}+\boldsymbol{v}_{3} \alpha_{3}, \\
& \zeta=\zeta_{1} \alpha_{1}+\zeta_{2} \alpha_{2}+\zeta_{3} \alpha_{3}, \\
& \lambda=\lambda_{1} \alpha_{1}+\lambda_{2} \alpha_{2}+\lambda_{3} \alpha_{3}, \\
& \rho=\rho_{1} \alpha_{1}+\rho_{2} \alpha_{2}+\rho_{3} \alpha_{3} .
\end{aligned}
$$

Здесь плотность тока $\boldsymbol{j}$, протекающего в ванне, с учетом индуцированных токов опишем следующим соотношением:

$$
\boldsymbol{j}=\sum_{m=1,2,3}\left(\alpha_{m} \sigma_{m}\right)\left(\mu_{0}[\boldsymbol{v}, \boldsymbol{H}]-\nabla \phi\right) .
$$

В начальный момент времени значения объемных долей, полей скоростей, температур и концентраций ионов считают известными. Граничные условия и постановка задачи полностью приведены в работе [5].

\section{2. Численный метод решения}

Для решения поставленной задачи применяется численный метод, основанный на расщеплении исходной задачи по физическим процессам [6]. Используется равномерная сетка по пространству, которая разбивает расчетный объем на прямоугольные параллелепипеды. Центрам масс параллелепипедов приписываются следующие скалярные величины: концентрации ионов, объемные доли, давление, электрический потенциал, температура; граням - потоковые величины: скорости фаз, плотность электрического тока, магнитный потенциал; на ребрах определяются вихревые величины: компоненты вектора напряженности магнитного поля $[7,8]$. Шаг по времени определяется из условия устойчивости схемы на каждом шаге [9].

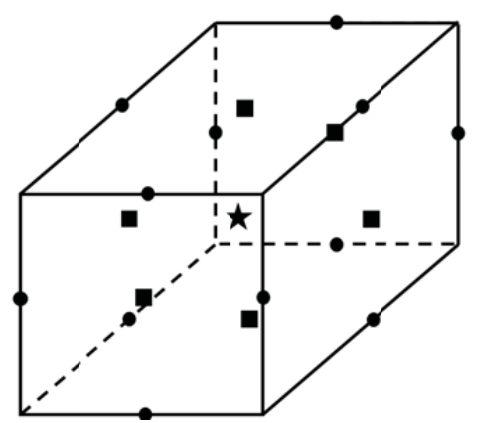

Рис. 1. Расчетная ячейка, - - центр граней (скорости фаз, плотность электрического тока, магнитный потенциал), - ребра (напряженность магнитного поля), „ - центр ячейки (концентрации ионов, объемные доли, давление, электрический потенциал, температура)

Fig. 1. Calculation cell, - the center of the faces (phase velocities, electric current density, magnetic potential), - - edges (magnetic field strength), $\star$ - cell center (ion concentration, volume fractions, pressure, electric potential, temperature) 
Таблица 2. Изменение объемного баланса смеси

Table 2. Change in volumetric balance of the mixture

\begin{tabular}{|c|c|}
\hline$t_{n}, c$ & $\left|1-\left(a_{1}+a_{2}+a_{3}\right)\right|$ \\
\hline 0,0 & 0,0 \\
\hline 0,1 & $0,1 \mathrm{e}-13$ \\
\hline 1,0 & $0,453 \mathrm{e}-11$ \\
\hline 10,0 & $0,293 \mathrm{e}-7$ \\
\hline 100,0 & $0,583 \mathrm{e}-6$ \\
\hline 110,0 & $0.353 \mathrm{e}-6$ \\
\hline
\end{tabular}

Дифференциальные уравнения аппроксимируются явными разностными схемами со вторым порядком по пространству и времени. Использование явных схем дает возможность распараллелить процесс решения, что ведет к сокращению времени расчета. Численное исследование свойств разностного метода решения на сгущающихся сетках показало, что суммарная аппроксимация дает первый порядок точности по пространству. При проведении численных экспериментов особое внимание уделяли сохранению объемного баланса смеси, уравнение которого явно не входит в систему дифференциальных уравнений, описывающих математическую модель. В табл. 2 приведены отклонения объемного баланса компонент смеси в процессе расчета во времени. Из анализа численных результатов видно, что со временем величина отклонения растет, но не превышает суммарной погрешности схемы. Результаты табл. 2 позволяют утверждать наличие сходимости предложенного численного решения с достаточной точностью. Проведенные тестовые расчеты для модельных задач и расчеты отдельных верификационных вычислительных экспериментов по моделированию магнитной гидродинамики подтвердили адекватность математической модели [5] и численной реализации представленного алгоритма [10].

\section{3. Анализ численных экспериментов}

Проведено математическое моделирование динамики границы раздела сред алюминий электролит в зависимости от распределения потенциала по аноду для электролизера Содерберга и многоанодного электролизера. Основными различиями в конструкции электролизеров являются количество анодов и технология их изготовления. При моделировании многоанодного электролизера был взят электролизер с 22-мя обожженными анодами, в электролизере Содерберга используется один самообжигающийся анод. Конфигурация анодов определяет электромагнитные поля в электролизерах, плотность протекающего тока и силу Лоренца. Условия сравнения по МГД-стабильности работы электролизеров приведены в работе [11]. Качественное распределение электромагнитного поля вблизи анодов на электролизере Содерберга и многоанодном электролизере изображено на рис. 2 и 3.

В ходе исследований было выявлено, что любое неравномерное распределение потенциала вызывает значительные возмущения на границе раздела фаз металл - электролит. В силу технологических особенностей добиться равномерного распределения потенциала по аноду прак-

$$
-248-
$$




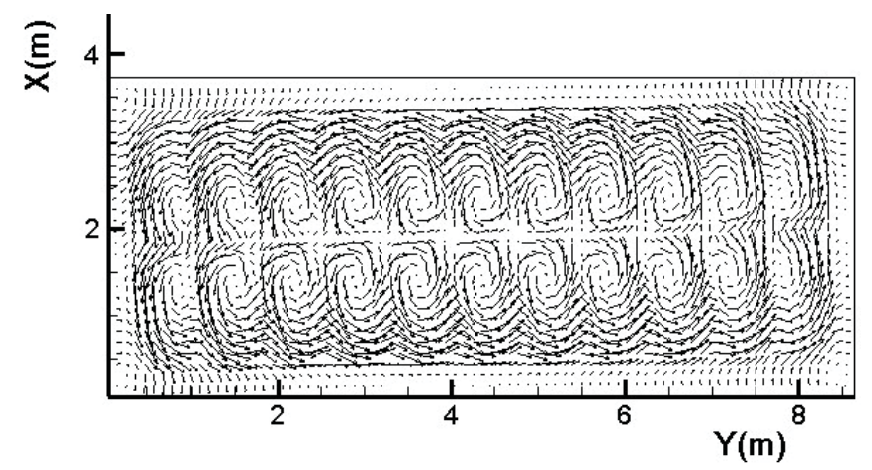

Рис. 2. Напряженность магнитного поля в плоскости XY (z=0.3 м) в многоанодном электролизере

Fig. 2. Magnetic field strength in the XY plane $(\mathrm{z}=0.3 \mathrm{~m})$ in a multi-anode cell

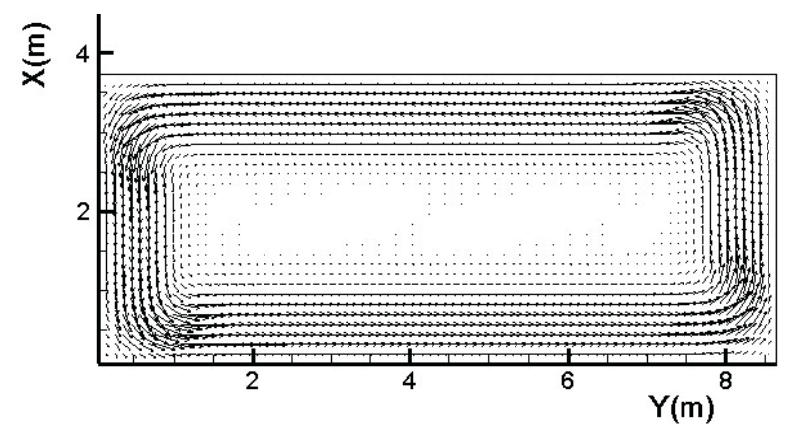

Рис. 3. Напряженность магнитного поля в плоскости XY (z=0.3 м) в электролизере Содерберга

Fig. 3. Magnetic field in the XY plane $(\mathrm{z}=0.3 \mathrm{~m})$ in the Soderberg electrolyzer

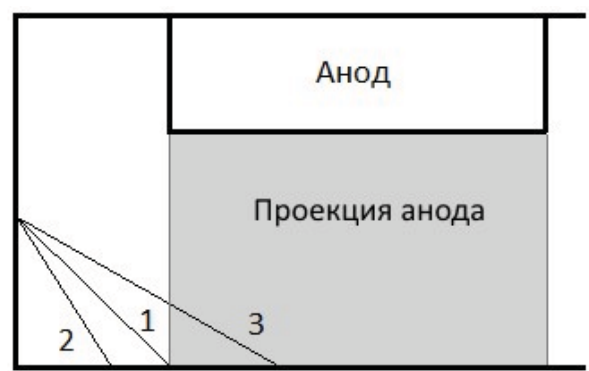

Рис. 4. Расположение настыли

Fig. 4. Accretion of sideledge

тически невозможно. Таким образом, проведенное численное исследование позволяет сделать вывод о том, что электролизер Содерберга менее МГД-стабилен, чем многоанодный электролизер с обожженными анодами.

Рассматривали задачу об изменении формы рабочего пространства ванны и его влияние на МГД-стабильность в многоанодном электролизере для различных начальных форм настыли. На рис. 4 показана форма настыли, а на рис. 5 представлен график расстояния между зоной

$$
-249-
$$




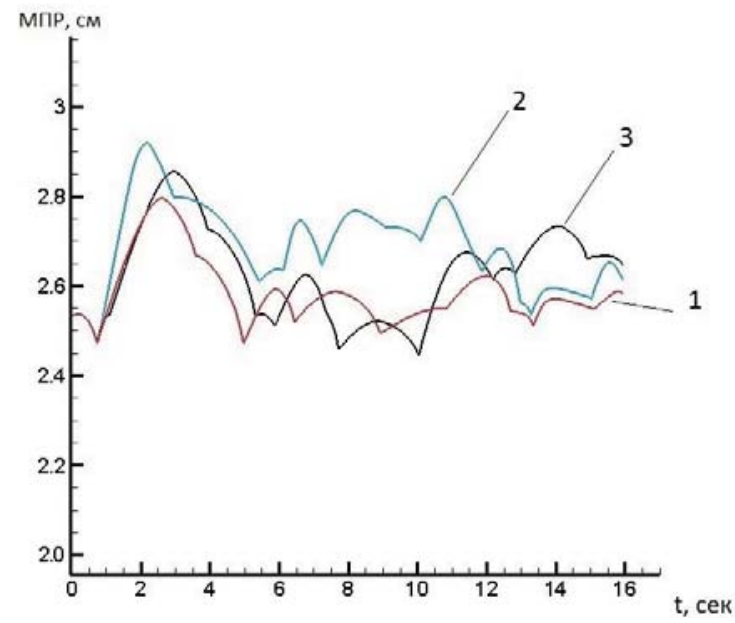

Рис. 5. График расстояния между зоной обратного окисления и границей зон металл - элетролит для разных начальных форм настыли

Fig. 5. Graph of the distance between the reverse oxidation zone and the boundary of the metal-electrolyte zones for different initial forms of accretion

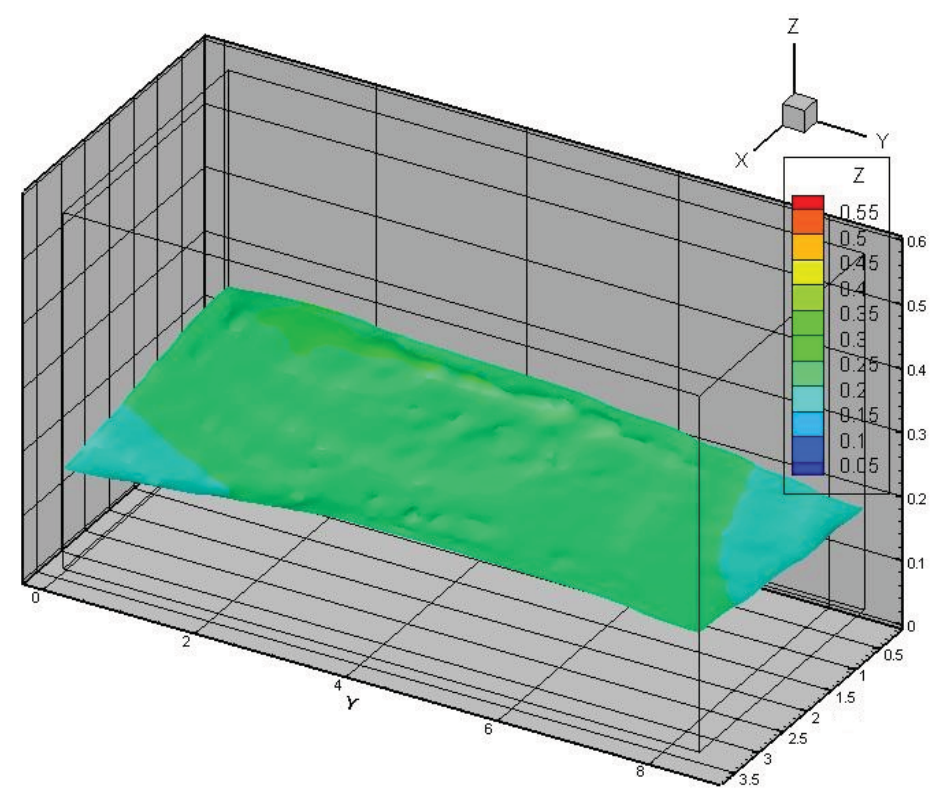

Рис. 6. Граница сред электролит - металл

Fig. 6. Electrolyte-metal media boundary

обратного окисления и границей зон металл - элетролит для случаев, когда настыль непосредственно под проекцией анода не доходит до проекции анода и заходит за проекцию анода первая, вторая и третья кривые соответственно.

Показано, что наиболее МГД-стабильная работа соответствует случаю, когда настыль подведена непосредственно под проекцию анодов на дно ванны. 
Были рассчитаны граница раздела сред электролит - металл, представленная на рис. 6, и граница зоны обратного окисления, которая определяется пространственным распределением газовой фазы.

На рис. 7 приведены температурные поля для рассматриваемых случаев расположения настыли. Как видим, с увеличением длины настыли температура на подине увеличивается, что объясняется увеличением плотности тока в этой области. Видно также, что с увеличением длины настыли температура внешних краев уменьшается, что объясняется тоже уменьшением плотности тока в этой области.

Технологическую опасность представляет собой расстояние между кривыми, достигающими критического значения расстояния между зоной обратного окисления и границей зон металл - элетролит, при котором возникает обратное окисление, что приводит к потере выхода металла по току. На рис. 8 прямой линией обозначена граница подошв анодов, верхней

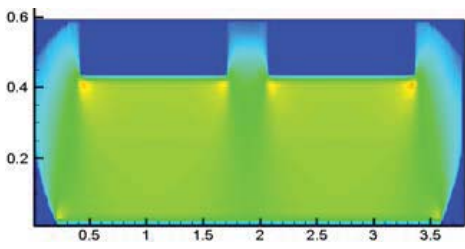

a

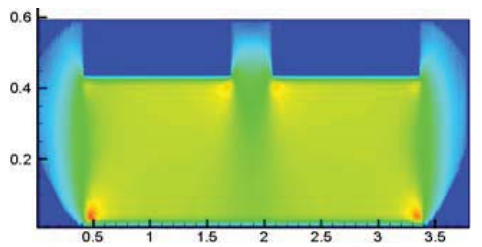

6

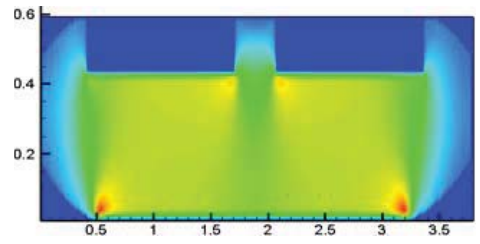

B

T: $945.1946 .6948 .1949 .6951 .1952 .6954 .1^{\circ} \mathrm{C}$

Рис. 7. Температурное поле: а - настыль не доходит до проекции анода; б - настыль непосредственно под проекцией анода; в - настыль заходит за проекцию

Fig. 7. Temperature field: $\mathrm{a}$ - sideledge does not reach the projection of the anode; $\sigma$ - sideledge directly under the projection of the anode; $\mathrm{B}$ - sideledge goes beyond the projection

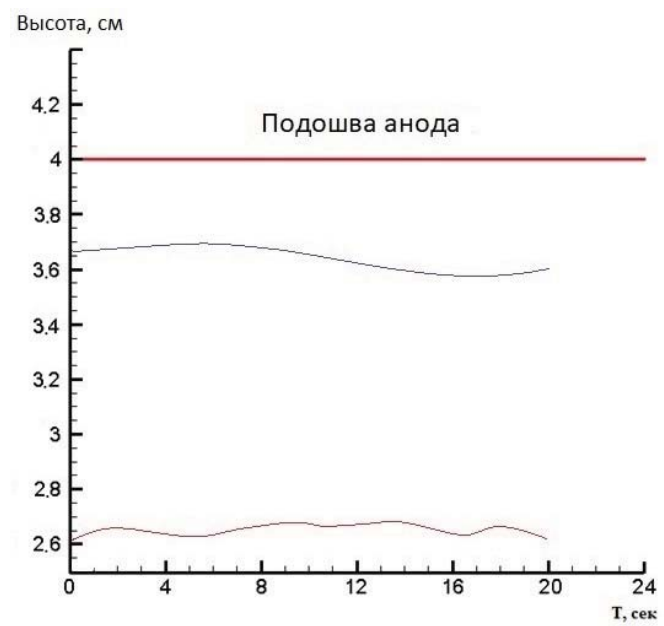

Рис. 8. График расстояния между зоной обратного окисления и границей зон металл - элетролит и нижней границы зоны обратного окисления при равномерном распределении потенциала по анодам

Fig. 8. Graph of the distance between the reverse oxidation zone and the boundary of the metal-electrolyte zones and the lower boundary of the reverse oxidation zone with a uniform distribution of potential across the anodes 
кривой - нижняя граница зоны обратного окисления, нижней кривой - граница жидкого алюминия.

Верификация полной математической модели была проведена на примере задачи о выемке крайней пары анодов (анодов 11 и 22) (см. [12, 13]). Данная постановка верификационной задачи характерна тем, что в процессе замены 11-го и 22-го анодов развивается МГД-нестабильность. Сравнение с известными результатами, полученными другими авторами при численном моделировании процесса электролиза при замене выгоревших анодов [14], дало удовлетворительные результаты.

В заключение отметим, что преимущество представленной модели состоит в учете и во взаимосвязи гидродинамических, электромагнитных, тепловых и электрохимических процессов, а также в учете изменения геометрии ванны за счет процессов тепломассопереноса, что позволяет проводить математическое моделирование различных технологических особенностей протекания промышленного электролиза алюминия.

\section{Список литературы / References}

[1] Нигматулин Р.И. Основы механики гетерогенных сред. М.: Наука, 1978. 336 с. [Nigmatulin R.I. Fundamentals of the mechanics of heterogeneous media. Moscow: Nauka, 1978. 336 p. (in Russian)]

[2] Ландау Л.Д., Лифшиц Е.М. Теоретическая физика. М.: Наука, 1982. Т.8. 621 с. [Landau L.D., Lifshits E.M. Theoretical Physics, Moscow: Nauka, 1982. Vol. 8. 621 p. (in Russian)]

[3] Ariana M., Desilets M., Proulx P. On the analysis of ionic mass transfer in the electrolytic bath of an aluminum reduction cell. The Canadian journal of chemical engineering, 2014, 92, 19511964.

[4] Burger M., Schlake B., Wolfram M.-T. Nonlinear Poisson-Nernst-Planck equations for ion flux through confined geometries. IOP Publishing Ltd \& London Mathematical Society Nonlinearity, 2012, 25, 4, 961-991.

[5] Калмыков. А.В. Математическое моделирование влияния проиессов тепломассопереноса на МГД-стабильность алюминиевого электролизера. Диссертация. Москва, 2017. [Kalmykov A.V.. Mathematical modeling of the influence of heat and mass transfer processes on the MHD stability of an aluminum electrolyzer. Thesis. Moscow, 2017 (in Russian)]

[6] Ковеня В.М., Слюняев А.Ю. Модификации алгоритмов расщепления для решения уравнений газовой динамики и Навье-Стокса. Вычислительные технологии. 2007, 12(3), 71-86. [Covenya V.M., Slyunyaev A.Yu., Modifications of splitting algorithms for solving equations of gas dynamics and Navier-Stokes. Computational technologies. 2007, 12(3), 71-86 (in Russian)]

[7] Патанкар С. Численные методы решения задач теплообмена и динамики жидкости. М.: Энергоатомиздат, 1984, 152 c. [Patankar S. Numerical methods for solving heat transfer problems and fluid dynamics. Moscow: Energoatomizdat, 1984. 152 p. (in Russian)]

[8] Nikitin N. Finite-difference method for incompressible Navier-Stokes equations in arbitrary orthogonal curvilinear coordinates. Journal of Computational Physics, 2006, 217, 759-781.

[9] Chase R., Gibson R., Marks J., PFC emissions performance for the global primary aluminum industry. Light metals, 2005, 279-282. 
[10] Анпилов С.В. Однофазные и многофазные математические модели электролиза алюминия. Диссертация. Москва, 2011. [Anpilov S.V. Single-phase and multiphase mathematical models of aluminum electrolysis. Thesis. Moscow, 2011 (in Russian)]

[11] Савенкова Н.П., Анпилов С.В., Калмыков А.В., Проворова О.Г., Пискажова Т.В. Сравнение результатов математического моделирования МГД-стабильности многоанодного алюминиевого электролизера и электролизера Содерберга. Математика. Компьютер. Образование: сб. тезисов ХХІ международной конференции. Под ред. Г.Ю. Ризниченко. М.-Ижевск, 2014. C. 101. [Savenkova N.P., Anpilov S.V., Kalmykov A.V., Provorova O.G., Piskazhova T.V. Comparison of the results of mathematical modeling of MHD stability of a multi-anode aluminum electrolyzer and Soderberg electrolyzer. Mathematics. A computer. Education: Sat abstracts of the XXI international conference / Ed. G.Yu. Riznichenko. M.-Izhevsk, 2014. P. 101 (in Russian)]

[12] Савенкова Н.П., Анпилов С.В., Кузьмин Р.Н., Проворова О.Г., Пискажова Т.В. Двухфазная 3D-модель МГД-явлений алюминиевого электролизера. Цветные металль - 2011», Раздел 3. Получения алюминия. Сборник докладов третьего международного конгресса. Красноярск, 2011, c. 282-286. [Savenkova N.P., Anpilov S.V., Kuzmin R.N., Provorova O.G., Piskazhova T.V. Twophase 3D model of MHD phenomena of an aluminum electrolyzer. Non-ferrous metals - 2011, Section 3 "Production of aluminum". Collection of reports of the third international congress. Krasnoyarsk, 2011, p. 282-286. (in Russian)]

[13] Савенкова Н.П., Анпилов С.В. Двухфазная трехмерная модель МГД-стабильности алюминиевого электролизера. Тихоновские чтения, сб. тезисов, М.: МАКС Пресс, 2011, с. 70-71. [Savenkova N.P., Anpilov S.V. Two-phase three-dimensional model of MHD stability of an aluminum electrolyzer. Tikhonov readings, Sat abstracts, M.: MAX Press, 2011, p. $70-71$ (in Russian)]

[14] Савенкова Н.П., Шобухов А.В., Анпилов С.В., Кузьмин Р.Н. Математическое моделирование физико-технологического процесса электролиза, Прикладная физика, 2009, 6, 43-51 [Savenkova N.P., Shobukhov A.V., Anpilov S.V., Kuzmin R.N. Mathematical modeling of the physical and technological process of electrolysis. Applied Physics, 2009, 6, 43-51 (in Russian)] 